\title{
Traumatic spinal injury and spinal cord injury: point for active physiological conservative management as compared to surgical management
}

\author{
W. S. El Masri(y) $)^{1,2}$
}

Received: 7 January 2018 / Accepted: 16 January 2018

(c) International Spinal Cord Society 2018

\begin{abstract}
The controversy about surgical vs conservative treatment of the injured spine with cord damage is centuries old. Until the end of the Second World War the majority of patients died or lived a short miserable life. Subsequently, Guttmann, an experienced neurosurgeon, realised surgery was not beneficial and sometimes detrimental to the person with spinal cord injury. Guttman, Frankel and others demonstrated with expert conservative management of the spine and the multi-system consequences of cord damage most patients made some neurological recovery and most with incomplete cord injury recovered ambulation regardless of X-ray findings. Attention to the non-medical effects of paralysis and post discharge supervision enabled persons with SCIs to enjoy complication free, dignified, productive and even competitive lives in sport and employment. The introduction of CT, MRI and safe anaesthesia led to the beliefs that surgical realignment, stabilisation and/or decompression improved neurologic outcome, facilitated early mobilisation and completion of rehabilitation, shortened hospitalisation, facilitated management and reduced cost. However, there is no supporting evidence for these claims. This manuscript describes the rationale and outcomes of conservative management and the weakness of the arguments for surgical management.
\end{abstract}

The intact spinal cord (SC) of patients with spinal injury (SI) does not sustain breaching of its blood brain barrier or loss of auto-regulatory mechanisms or cell membrane disturbances. It is therefore physiologically stable and only at risk of damage from poorly managed biomechanical instability (BI) of the injured spine with inadequate nonoperative or surgical management. When the SC is injured it loses these protective physiological functions, becomes physiologically unstable and at risk of further damage from avoidable medical complications from related changes in other body systems. [1, 2]. Generalised sepsis from the urinary tract, lungs, bones or skin, blood pressure (BP) instability, hypoxia, poikilothermia, electrolyte imbalance, and abdominal distension resulting in inadequate breathing/ oxygenation can all cause temporary or permanent

\footnotetext{
W. S. El Masri(y)

bellstonehse@btinternet.com

Keele University, Keele, UK

2 Midland Centre for Spinal Injuries, Robert Jones \& Agnes Hunt Orthopaedic Hospital, Oswestry, UK
}

neurological deterioration or lack of recovery [1-3]. A person with spinal cord injury (SCI) is therefore at a higher risk of neurological deterioration than the neurologically intact patient from both the physiological instability of the injured SC as well as the BI of the injured spinal axis.

Over the last 70 years reports have demonstrated Active Physiologic Conservative Management (ACPM) yields excellent neurological outcomes [3-5]. APCM requires simultaneous attention to the injured spine as well as all systems affected by SCI, in recumbence from hours post injury for between 4-6 weeks. Subsequently, a similar period of bracing is necessary when the individual is mobilised. Recumbence is necessary during this time when vaso-motor and locomotor reflexes are absent or weak with subsequent risk of postural hypotension with decreased vital capacity (VC), oxygen saturation and hypoxia during verticalisation. During recumbence BI is contained by external orthosis. The patient is log rolled every $2-3 \mathrm{~h}$. Along with anticoagulation, the legs are slightly elevated to prevent pooling of venous blood, increased risk of deep vein thrombosis and pressure sores on the heels. Intensive noninvasive respiratory care is commenced to prevent chest infections and hypoxia. Forty-eight hours post injury, a 
bladder regime of four hourly intermittent catheterisation and bowel programme is used to achieve continence and prevent constipation. Passive range of movement is used to maintain range of motion and precent muscle contractures. Active exercise of unaffected muscles is encouraged with repeated shoulder positioning in abduction to preserve shoulder range of movement. The patient receives education, counselling and peer support.

Patients with cervical injury are likely to require traction, assisted coughing and forearm and hand elevation on pillows to prevent oedema and swelling of the hands and fingers. Before mobilisation, tilt table studies with monitoring of BP, VC $[6,7]$ and neurologic function every $10^{\circ}$ of incline are performed until the patient reaches $80^{\circ}$ in incline, following which the patient is fully mobilised. Once fully mobilised in the wheelchair, the patients actively participates in locomotor and psycho-social rehabilitation and continues education in self-care and prevention skills in conjunction with attention to various body systems and the aforementioned treatments.

With APCM $20-30 \%$ of all persons presenting within 2 weeks of SCI with complete injury improve 1-2 Frankel grades or more. About 50\% of persons presenting with clinical complete cauda equina will walk again. Between 50 and $70 \%$ of patients with only distal sensory sparing recover walking and 75 to $80 \%$ of patients with insignificant distal motor sparing recover to walk again $[4,5]$. Motor recovery has been consistently shown to return in the myotomes corresponding to the dermatomes with spino-thalamic sensory sparing (STSS) in patients with both complete and incomplete cord injury [8]. We hypothesised this is due to the proximity between the spino-thalamic and corticospinal tracts, suggesting that sparing in the former may also indicate some sparing in the later $[8,9]$ and this was confirmed by other groups [10-12]. Those patients with complete cord injury but spared STSS in the zone of partial preservation recover motor power in the dermatomes corresponding to STSS sparing [9]. Over $75 \%$ of patients with traumatic SCI, who present in the first $48 \mathrm{~h}$ of injury with complete motor loss but SSTC down to S2 treated with APCM walk again [12]. To date there is no credible evidence that by realigning the spinal axis, surgically stabilising the spine, correcting these deformities, or decompressing the SC achieves equality or superiority of outcomes.

The current surgical opinion is that surgery gives better neurological results in patients presenting with incomplete than with complete injuries with little or no consideration that the neurological recovery may be even better if no intervention was carried out. The clinical prognostic indicators seem to be ignored in favour of radiological appearances (X-rays, computed tomography (CT), magnetic resonance imaging (MRI)) and a wide range of non- validated classifications, with assumption that $\mathrm{BI}$ is more safely and easily managed by surgical stabilisation.

The belief that the secondary cellular, enzymatic, chemical and other changes in the SC are minimised with surgical decompression to improve neurologic recovery in humans similar to rodents has led to the belief that humans must therefore benefit from surgical decompression. Unfortunately, this assertion ignores species differences, differences in physiological responses, in vivo versus in vitro models of SCI and differences in ease and reliability of assessment of neurological outcomes in the two species and that humans are bipeds, not quadrupeds. Moreover, the assertion has followed that it is possible that early rather than late surgery may give better neurological outcomes $[13,14]$, thus early surgery must therefore give better outcomes than APCM!

In SCI following APCM, there is significant neurological recovery irrespective of the severity of malalignment, BI, canal encroachment and/or cord compression without surgical realignment, stabilisation or decompression $[2,5,9$, 12, 15-22]. Most patients with incomplete SCI recover neurologically to walk without intervention (surgical, pharmacological, biological or cellular) for the primary or secondary injury. Biomechanical stability of the most unstable injuries is restored with the shortest fusion and the majority of patients regain a painless spine with excellent range of motion essential for independence. Furthermore, the spinal canal has been known to remodel with resorption of encroaching bone [23]. Therefore, any therapeutic intervention should have demonstration of evidence of equality or superiority of neurological and other relevant outcomes over those of APCM; however, this requires well designed trials with independent assessment of preintervention neurological status and outcomes.

With adequate APCM, permanent neurological deterioration is extremely rare in recumbence [24]. Temporary ascent of the level by one or two segments occurs in up to $9 \%$ of patients with complete SCI at about $48 \mathrm{~h}$ from injury and returns to or distal to the original level in almost all patients within 12 weeks. This is thought to be due to temporary cord oedema [9].

It is alleged that the incidence of post-traumatic syringomyelia (PTS) is lower in surgically managed patients than with non-operative management. Unfortunately, neither patient selection nor non-operative management is adequately described in these publications. The Oswestry Group published the incidence of PTS in 815 consecutive patients with up to 34 years follow-up (mean 8.6 years). The incidence of PTS ranged between 1.2 and $5.6 \%$ (mean $3.8 \%$ ) depending on the site and completeness of the injury [25]. This incidence is lower than any subsequently published incidence of PTS following surgery. 
Perioperative neurological deterioration after SCI can occur in the best surgical hands and the mechanism is poorly understood. Unfortunately, literature on the incidence and mechanisms of damage is scarce. Furthermore, there is no literature on the incidence of unfulfilled recovery because of intervention. In our experience neurological deterioration and lack of expected recovery do not necessarily always occur because of mechanical damage of the cord during surgery. Peri- and post-operative neurological deterioration and lack of neurological recovery can be attributed to ischaemia to the SC through hypotension or hypoxia during anaesthesia or perioperative ischaemia, post-operative epidural or subdural bleeding, early postoperative implant failure and post-operative sepsis. An increase of cerebrospinal fluid pressure, at and below the level of surgical decompression with possible reduction of SC perfusion has been demonstrated by Kwon et al. [26] and may also be a mechanism of neurological deterioration or lack of expected recovery following surgical decompression. Research is required to determine the exact incidence and cause of neurological deterioration following all methods of treatment.

APCM does not consider early mobilisation (EM) to be beneficial or safe in all patients with SCI. Therefore 4-6 weeks in recumbence and tilt table studies are performed prior to wheelchair mobilisation. There is no evidence that EM achieves more rapid completion of equal end points of rehabilitation, shorter hospitalisation or ease of nursing care. Moreover, due to the complex pathophysiological effects of cord damage and their management, the opposite is likely to be true.

The undoubted psychological and economic benefits of early mobilisation of the neurologically intact person cannot be extrapolated to the person with SCI who requires all the energy, maintenance of BP and oxygen saturation possible to participate fully in rehabilitation. Patients often feel poorly physically and psychologically, are unable to actively participate in intensive physical rehabilitation, and often repeatedly request to go back to bed until all their vaso-motor and somatic reflexes return.

The aims of APCM are more than the protection of the SC until restoration of biomechanical stability. It is as important to maximise neurological recovery, minimise pain and maximise range of motion of the spine, achieve safe and convenient functioning of each of affected body system as well as enable the patient to find a useful and enjoyable life in the community. All these aims are achievable at a low cost with APCM and with adequate monitoring post discharge. Furthermore, the absence of implants or fragments of removed implants will enable future researchers to use advanced imaging techniques to visualise cord regeneration and evaluate new interventions that may be beneficial.
Most persons with SCI can be managed without surgery; however, surgical indications remain including spinal injury without neurologic damage or ligamentous injuries without bony injury. The wish of patients who make an evidencedbased informed choice of surgery and EM with full knowledge of the potential hazards should be respected. Persons with uncontrolled epilepsy, behavioural problems and inability to comply with APCM and patients with neurological deterioration with signs of further cord compression on MRI and loss of pin prick sensation are also appropriate for surgery.

\section{Conclusion}

The foundation of the APCM school of thought is persons with SCI are at higher risk of neurological deterioration or lack of recovery with surgery from para-surgical mishaps and possibly decreased SC perfusion during surgery. Surgery is beneficial for the neurologically intact and a minority of neurologically impaired patients. However, there is no credible evidence that in persons with SCI cutting through nerve endings, dissecting traumatised soft tissue to access the spine, or manipulating damaged bony tissue to implant and/or decompress the spine results in any added value neurologically, economically or otherwise. Surgery and EM have not shown equal or better neurological outcomes, complication rates, level of independence, equal or shorter recumbence, total hospitalisation days, lower hospital readmission rates or costs of hospitalisation or long term care. In light of this, persons with SCIs should be given an informed, evidence-based choice about whether or not to undergo surgery. When surgery is indicated it is best carried out in spinal injury centres where the expertise and infrastructure are available to provide a fit for purpose care that is not extrapolated from the neurologically intact patient.

\section{Compliance with ethical standards}

Conflict of interest The authors declare that they have no conflict of interest.

\section{References}

1. El Masri (y) WS. Physiological instability of the spinal cord following injury. Paraplegia. 1993;31:273-75.

2. El Masri (y) WS. Traumatic spinal cord injury: the relationship between pathology \& clinical implications. Trauma. 2006;8:29-46.

3. Guttmann L. Spinal cord injuries: comprehensive management and research. 2nd ed. Oxford: Blackwell; 1976.

4. Frankel HL, Hancock DO, Hyslop G, Melzack J, Michaelis LS, Ungar $\mathrm{GH}$, et al. The value of postural reduction in initial 
management of closed injuries of the spine with paraplegia and tetraplegia. Paraplegia. 1969;7:179-92.

5. El Masri(y) WS, Jaffray DJ. Recent developments in the management of injuries of the cervical spine. In: Frankel HL, editor. Spinal cord trauma. Amsterdam: Elsevier; 1992. p. $55-73$.

6. Kumar N, Pieri-Davies S, Chowdhury JR, Osman A, El Masri (y) WS. Evidence-based respiratory management strategies required to prevent complications and improve outcome in acute spinal cord injury patients. Trauma. 2017;19:23-29.

7. Morgan MDL, Silver JR, Williams SJ. The respiratory system of the spinal cord patient. In: Bloch RF, Basbaum M editors. Management of spinal cord injury. Baltimore: Williams and Wilkins; 1986. p. 78-17.

8. Folman Y, El Masri (y) WS. Spinal cord injury: prognostic indicators. Injury. 1989;20:92-3.

9. Katoh S, El Masri (y) WS. Neurological recovery after conservative treatment of cervical cord injuries. J Bone Joint Surg Br. 1994;76B:225-28.

10. Crozier KS, Graziani V, Ditunno JF Jr, Herbison GJ. Spinal cord injury: prognosis for ambulation based on sensory examination in patients who are initially motor complete. Arch Phys Med Rehabil. 1991;72:119-21.

11. Poynton AR, O' Farrell DA, Shannon F, Murray P, McManus F, Walsh MG. Sparing of sensation to pin prick predicts recovery of a motor segment after injury to the spinal cord. J Bone Jt Surg Br. 1997;79:952-4.

12. Katoh S, El Masry WS, Jaffray D, McCall IW, Eisenstein SM, Pringle RG, et al. Neurologic outcome in conservatively treated patients with incomplete closed traumatic cervical spinal cord injuries. Spine. 1996;21:2345-51.

13. Van Middendorp JJ. Letter to the editor: "Early versus delayed decompression for traumatic cervical spinal cord injury: results of the Surgical Timing in Acute Spinal Cord Injury Study (STASCIS)". Spine J. 2012;12:540-2.

14. Fehlings MG, Vaccaro A, Wilson JR, Singh A, W Cadotte D, Harrop JS, et al. Early versus delayed decompression for traumatic cervical spinal cord injury: results of the Surgical Timing in Acute
Spinal Cord Injury Study (STASCIS). PLoS ONE. 2012;7: e32037.

15. El Masri WS, Meerkotter DV. Early decompression of the spinal cord following injury: arguments for and against. In: Ellis LSeditor. Spinal cord dysfunction. Vol II Intervention \& treatment. Oxford: Oxford University Press; 1992. p. 7-27.

16. Rosenberg N, Lenger R, Weisz I, Stein H. Neurological deficit in a consecutive series of vertebral fractures patients with bony fragments within the spinal canal. Spinal Cord. 1996;35:92-5.

17. El Masri(y) WS, Short DJ. Current concepts: spinal injuries \& rehabilitation. Curr Opin Neurol. 1997;10:484-92.

18. Boerger TO, Limb D, Dickson RA. Does canal clearance affect neurological outcome after thoracolumbar burst fractures. J Bone Jt Surg Br. 2000;82B:629-35.

19. Mohanty SP, Venkatram N. Does neurological recovery in thoracolumbar and lumbar burst fractures depend on the extent of canal compromise? Spinal Cord. 2002;40:295-99.

20. El Masry WS, Osman AE. Clinical perspectives on spinal injuries. In: Cassar-Pullicino V, Imhof $\mathrm{H}$, editors. Spinal trauma: an imaging approach. New York, NY: Thieme Medical; 2006. p. 1-14.

21. Ibrahim A, Li Y, Li D, Raisman G. Olfactory ensheathing cells: ripples of an oncoming tide?. Lancet Neurol. 2006;5:453-57.

22. El Masri(y) WS, Kumar N. Active physiological conservative management in traumatic spinal cord injuries-an evidence-based approach. Trauma. 2017;19:10-22.

23. Fidler MW. Re-modelling of the spinal canal after burst fracture. A prospective study of two cases. J Bone Joint Surg. 1988;70B:730-32.

24. Marshall LF, Knowlton S, Garfin SR, Klauber MR, Eisenberg HM, Kopaniky D, et al. Deterioration following spinal cord injury: a multicenter study. J Neurosurg. 1987;60:400-4.

25. El Masri(y) WS, Biyani A. Incidence, management and outcome of post traumatic syringomyelia. In memory of Mr Bernard Williams. J Neurol Neurosurg Psychiatry. 1996;60:141-6.

26. Kwon BK, Curt A, Belanger LM, Bernardo A, Chan D, Markez $\mathrm{JA}$, et al. Intrathecal pressure monitoring and cerebrospinal fluid drainage in acute spinal cord injury: a prospective randomized trial. J Neurosurg Spine. 2009;10:181-93. 